\title{
Synthesis, characterization, antituberculosis activity and computational studies on novel Schiff bases of 1,3,4- thiadiazole derivatives
}

\author{
Sevda TÜRK 1 (D), Sevgi KARAKUŞ $2 *$ (D), Arooma MARYAM 3,4 (D), Emine Elçin ORUÇ-EMRE 5 (D) \\ 1 Department of Pharmaceutical Chemistry, Faculty of Pharmacy, Karadeniz Technical University, Ortahisar 61080 \\ Trabzon, Turkey. \\ 2 Department of Pharmaceutical Chemistry, Faculty of Pharmacy, Marmara University, Başıbüyük 34854 İstanbul, \\ Turkey. \\ 3 Department of Pharmaceutical Chemistry, Faculty of Pharmacy, Biruni University, Topkap1 34010 İstanbul, Turkey. \\ 4 Department of Biosciences, COMSATS University Islamabad, Pakistan. \\ 5 Department of Chemistry, Faculty of Arts and Sciences, Gaziantep University, Şehitkamil 27310 Gaziantep, Turkey. \\ * Corresponding Author. E-mail: skarakus@marmara.edu.tr (S.K.); Tel. +90-216-414 2962.
}

Received: 24 July 2020 / Revised: 14 September 2020/ Accepted: 17 September 2020

\begin{abstract}
A series of novel Schiff bases were designed and synthesized by the condensation of 1,3,4-thiadiazoles that contain aromatic primary amine and variously substituted benzaldehydes. The synthesized compounds were screened for their antituberculosis activity against Mycobacterium tuberculosis $H_{37} R v$ using BACTEC 460 radiometric system. Among the tested compounds, 2-(4-nitrophenyl)amino-5-[4-(3-(4-phenoxy))benzylideneaminophenyl]-1,3,4thiadiazole (3n) showed the highest inhibitory activity $(80 \%)$. The activities of the newly synthesized Schiff bases were higher in comparison to those of intermediate products 2-(4-aminophenyl)-5-aryl/alkylamino-1,3,4thiadiazoles (2a-1). The computational studies were also performed to estimate drug-like profile of the compounds by using QikProp analysis.
\end{abstract}

KEYWORDS: Schiff bases; 1,3,4-thiadiazoles; antituberculosis activity; Mycobacterium tuberculosis $\mathrm{H}_{37} \mathrm{Rv}$; ADME.

\section{INTRODUCTION}

Tuberculosis (TB) is known as an infectious disease, especially monitored on lungs, caused by a group of Mycobacterium species, mostly by Mycobacterium tuberculosis [1]. It is informed that among the world, every one of three people comes face to face with latent tuberculosis. And, one-tenth of latent tuberculosis turns into active TB disease [2]. With respect to the WHO's Global Tuberculosis Report 2019, TB is one of the important causes of death worldwide. Additionally, among the single infectious agents, it is more dangerous than HIV/AIDS. Furthermore, drug resistant TB continues to threaten public health, particularly in developing countries [3, 4]. Especially, for the treatment of multidrug resistant (MDR) TB, extremely drug resistant (XDR) TB and TB-HIV co-infections, the discovery of new antituberculosis agents is a necessity [5].

Schiff bases are important organic structures bearing $C=N$ linkage, the nitrogen atom linked to an alkyl or aryl moiety. They were firstly synthesized by Hugo Schiff at 1864 and since then they are called as Schiff bases. They are obtained by the condensation of primary amines with aldehydes or ketones [6-8]. Although mostly being distinguished by their antibacterial and antimicrobial activities, they also have come to the forefront by their antitubercular, antifungal, antioxidant, anti-inflammatory, antiviral and anticonvulsant activities [9-14]. Among this group, Terizidone is an important example to bacteriostatic agents bearing Schiff base structure, used in multidrug-resistant tuberculosis [15].

1,3,4-Thiadiazole is also a potent scaffold taking part in many drugs with various activities such as acetazolamide, methazolamide as CAs inhibitor; cefazolin, cefazedone as antimicrobial; megazole as antimicrobial and tripanoside etc. Besides their different pharmacological activities, 1,3,4-thiadiazoles exhibit remarkable antitubercular activity [16-21].

In the view of such information, we have combined these two active moieties with the aim to obtain new antitubercular agents. Besides elemental analysis, the newly synthesized compounds were characterized by various spectroscopic methods.

How to cite this article: Türk S, Karakuş S, Maryam A, Oruç-Emre EE. Synthesis, characterization, antituberculosis activity and computational studies on novel Schiff bases of 1,3,4-thiadiazole derivatives. J Res Pharm. 2020; 24(6): 793-800. 


\section{RESULTS AND DISCUSSION}

\subsection{Chemistry}

The novel compounds (3a-n) were synthesized according to the sequence shown in Figure 1. Firstly, 1,3,4-thiadiazoles (2a-n) were obtained by a four step reaction, starting from benzocaine, according to our previous reports $[18,22]$. Secondly, the Schiff bases (3a-n) were obtained by refluxing the (2a-n) compounds with various aldehydes, in methanolic medium over 45 minutes [23].

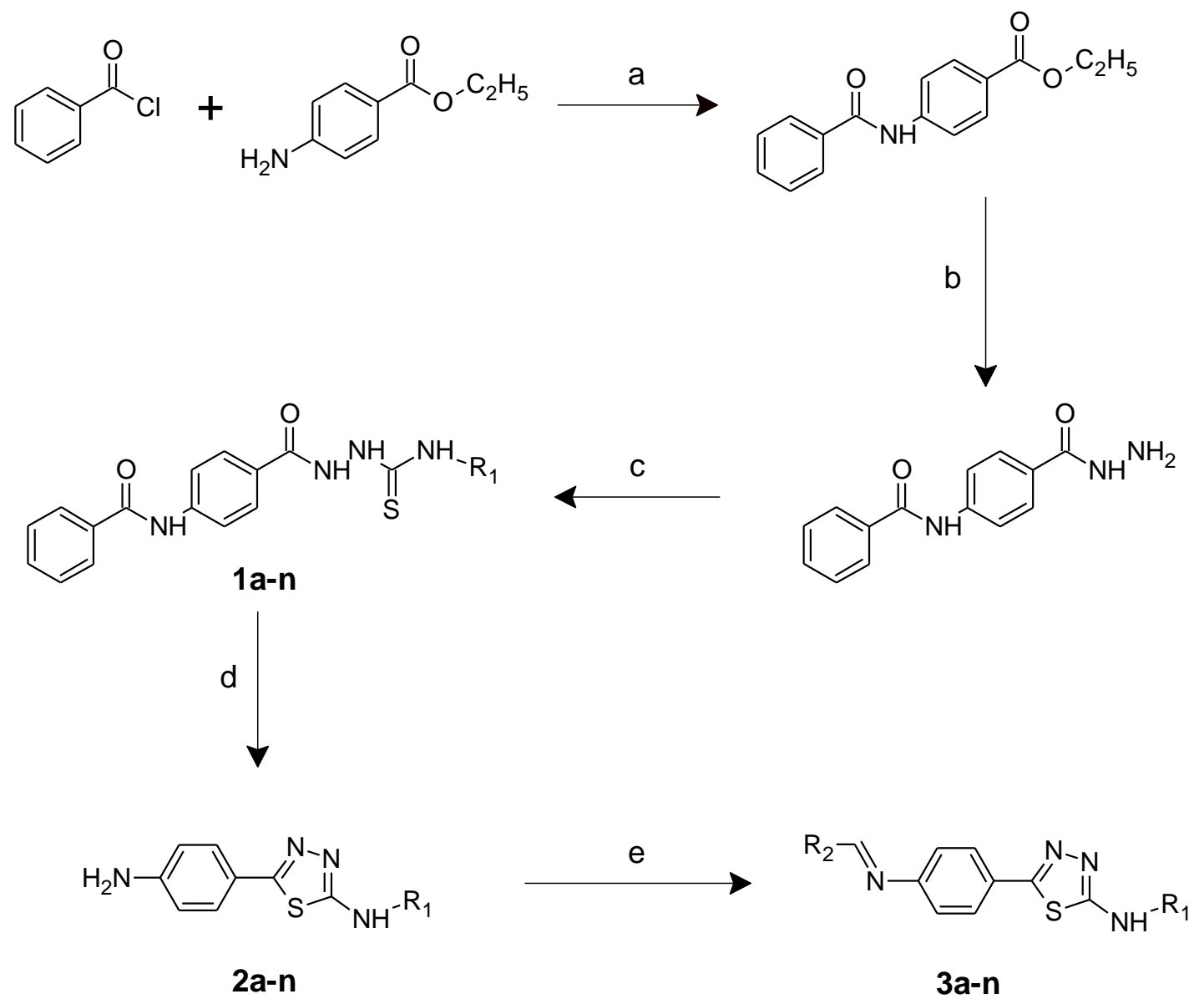

Figure 1. Synthetic pathway for compounds 3a-n. Reagents and conditions: a: Diethyl ether; b: $\mathrm{NH}_{2} \mathrm{NH}_{2} . \mathrm{H}_{2} \mathrm{O}, \mathrm{C}_{2} \mathrm{H}_{5} \mathrm{OH}$; c: $\mathrm{R}_{1}-\mathrm{NCS}, \mathrm{C}_{2} \mathrm{H}_{5} \mathrm{OH} ; \mathrm{d}$ : $50 \% \mathrm{H}_{2} \mathrm{SO}_{4}, \mathrm{NaOH}$; e: $\mathrm{R}_{2}-\mathrm{CHO}, \mathrm{CH}_{3} \mathrm{OH}$.

The structures of the newly synthesized 3a-n compounds were confirmed by FT-IR, ${ }^{1} \mathrm{H}-\mathrm{NMR}$ and mass spectroscopic methods, besides elemental analysis. The spectral data was in agreement with the proposed structures. According to the FT-IR spectra; the O-H stretching bands were screened at $3300-3390 \mathrm{~cm}^{-1}$ while the $\mathrm{N}-\mathrm{H}$ stretching bands were screened at $3150-3259 \mathrm{~cm}^{-1}$. Also, $\mathrm{C}=\mathrm{N}$ and $\mathrm{C}-\mathrm{Cl}$ stretching bands were screened between 1614-1643 $\mathrm{cm}^{-1}$ and $1072-1099 \mathrm{~cm}^{-1}$, respectively.

According to the ${ }^{1} \mathrm{H}-\mathrm{NMR}$ spectra; the $\mathrm{CH}=\mathrm{N}$ protons resonated at $8.69-10.53 \mathrm{ppm}$. In comparison with our previous study, the newly synthesized 3a-n compounds were thought to be in E-isomer form [24]. Although, the phenolic protons of compounds $\mathbf{3 a}-\mathbf{c}, \mathbf{3 e}, \mathbf{3 h}$, and $\mathbf{3 k}$ were detected at $14.11-14.40 \mathrm{ppm}$, as similar to the literature $[22,25]$, the phenolic proton of compound 31 couldn't been determined at the spectrum. The thiadiazole $-\mathrm{NH}$ - protons were detected as singlets at 10.39-11.32 ppm, and also the aromatic protons were observed at the expected regions.

According to the mass spectroscopic fragmentation of the compounds, which were studied under Atmospheric Pressure Chemical Ionization (APCI) positive polarity, the $(\mathrm{M}+\mathrm{H})^{+}$peaks were identified in agreement with their molecular structure. According to the mass spectra, in consequence of the cleavage of imine group, amine and substituted methylene fragments were occurred. 


\subsection{Antituberculosis activity}

Results of antituberculosis activity of the compounds are given in Table 1 and Table 2. The inhibition values of the synthesized compounds were found between 59-80\% compared to rifampicin. These values could be considered as a significant increase with reference to the intermediate products, 2a-1. For example, the 3eg compounds derived from 2e (having 4-fluorophenyl), showed 63-65\% inhibition. However, the 2e compound revealed only 3\% inhibition. Similarly, 2k (bearing 4-methoxyphenyl), the precursor of the $3 \mathbf{k}$ compound, showed only $7 \%$ inhibition. Whereas the $3 \mathrm{k}$ presented $69 \%$ inhibitory activity.

Table 1. Antituberculosis activity results of compounds 2a-1 [17].

\begin{tabular}{clcc}
\hline Compounds & $\mathbf{R}_{\mathbf{1}}$ & MIC $(\boldsymbol{\mu g} \mathbf{~ m l} \mathbf{- 1})$ & Inhibition $\%$ \\
\hline $\mathbf{2 a}$ & methyl & $>6.25$ & 29 \\
$\mathbf{2 b}$ & ethyl & $>6.25$ & 34 \\
$\mathbf{2} \mathbf{c}$ & cyclohexyl & $>6.25$ & 41 \\
$\mathbf{2 d}$ & benzyl & $>6.25$ & 37 \\
$\mathbf{2} \mathbf{e}$ & 4-fluorophenyl & $>6.25$ & 3 \\
$\mathbf{2 h}$ & 4-methylphenyl & $>6.25$ & 22 \\
$\mathbf{2 k}$ & 4-methoxyphenyl & $>6.25$ & 7 \\
$\mathbf{2}$ & 4-nitrophenyl & $>6.25$ & 37 \\
\hline Rifampicin & - & 0.25 & 98 \\
\hline
\end{tabular}

As a result, the antituberculosis activities of the newly synthesized Schiff bases seemed to be higher than the intermediate 2-substitutedamino-5-(4-aminophenyl)-1,3,4-thiadiazoles. The biological activity results indicated that compound 3n having phenoxy substituent attached to the 4-nitrophenylthiadiazole ring showed the highest inhibition with $80 \%$ inhibition value against Mycobacterium tuberculosis H37Rv at concentration of $6.25 \mu \mathrm{g} \mathrm{ml}^{-1}$.

\subsection{Drug-likeliness analysis}

With exception to Lipinski RO5, predicted values for total solvent accessible surface area (SASA), cell permeability (QPPMDCK), percentage of human absorption (HOA\%), blood brain barrier coefficient (QlogP $\mathrm{BB}$ ) and polar surface area (PSA) values calculated for 14 molecules understudy are satisfying the druglikeness criteria (Table 3). For SASA, recommended value for a molecule with good drug likeliness property should be in the range of 300.0 - 1000.0 while recommended values for Predicted brain/blood partition (QlogP BB) coefficient should be within the range of -3.0 - 1.2 [26]. In case of QikProp predicted permeability through the monolayers of Madin-Darby Canine Kidney cells (QPPMDCK), past studies reported that ligands having $<25 \mathrm{~nm} / \mathrm{sec}$ score have poor MDCK cell permeability whereas ligands bearing $>500 \mathrm{~nm} / \mathrm{sec}$ QPPMDCK value shows great potential of being a drug-like molecule. Similarly, human oral absorption (HOA\%) count in term of percentage should be preferably higher than $25 \%$ and to assess the bioavailability, polar absorption area of drug like compounds with $\leq 140 \AA^{2}$ value are considered good for drug optimization [27].

All the predicted drug likeliness parameters for 14 compounds given in Table 3 were within the recommended ranges/values. This indicate that these molecules are theoretically strong druggable candidates. Except compound $\mathbf{3 a}$ and compound $\mathbf{3 b}$, all compounds are non-complaint to Lipinski RO5. Although it is a drug-likeness guideline but there are so many Food and Drug Administration (FDA) approved drugs that don't follow Lipinski RO5 [28]. Drug-likliness has now thought to be linked with the dynamic nature of the chemical compound in which each possible conformation exhibits a distinct pattern of hydprophobicity, lipophilicity and molecular electrostatic potential (MEF).

\section{CONCLUSION}

Novel derivatives of Schiff bases were obtained from substituted 1,3,4-thiadiazoles. All of the final compounds were evaluated for their in vitro antituberculosis activity against Mycobacterium tuberculosis H37Rv. The activity results revealed that 2-(4-nitrophenyl)amino-5-[4-(3-(4-phenoxy))benzylideneaminophenyl]1,3,4-thiadiazole $3 \mathbf{n}$ was the most active compound by $80 \%$ inhibition value. According to the in silico calculations, all the synthesized compounds exhibit promising physicochemical properties, thus regarded as potential drug-like compounds for the discovery of therapeutic solutions against Mycobacterium tuberculosis infections. In this context, compound $3 \mathbf{n}$ could be used as a lead compound for further studies. 
Table 2. Antituberculosis activity results of compounds 3a-n.

\begin{tabular}{cllcc}
\hline Compounds & $\mathbf{R}_{\mathbf{1}}$ & $\mathbf{R}_{\mathbf{2}}$ & $\mathbf{M I C}(\boldsymbol{\mu g} \mathbf{~ m l} \mathbf{- 1})$ & Inhibition\% \\
\hline $\mathbf{3 a}$ & methyl & 2-hydroxy-3,5-dichlorophenyl & $>6.25$ & 69 \\
$\mathbf{3 b}$ & ethyl & 2-hydroxy-3,5-dichlorophenyl & $>6.25$ & 68 \\
$\mathbf{3 c}$ & cyclohexyl & 2-hydroxy-3,5-dichlorophenyl & $>6.25$ & 72 \\
$\mathbf{3 d}$ & benzyl & 2,4-dichlorophenyl & $>6.25$ & 61 \\
$\mathbf{3 e}$ & 4-fluorophenyl & 2-hydroxy-3,5-dichlorophenyl & $>6.25$ & 65 \\
$\mathbf{3 f}$ & 4-fluorophenyl & 2,4-dichlorophenyl & $>6.25$ & 63 \\
$\mathbf{3 g}$ & 4-fluorophenyl & 3,4-dichlorophenyl & $>6.25$ & 63 \\
$\mathbf{3 h}$ & 4-methylphenyl & 2-hydroxy-3,5-dichlorophenyl & $>6.25$ & 72 \\
$\mathbf{3 i}$ & 4-methylphenyl & 2,4-dichlorophenyl & $>6.25$ & 62 \\
$\mathbf{3 j}$ & 4-methylphenyl & 3-phenoxyphenyl & $>6.25$ & 73 \\
$\mathbf{3 k}$ & 4-methoxyphenyl & 2-hydroxy-3,5-dichlorophenyl & $>6.25$ & 69 \\
$\mathbf{3 1}$ & 4-nitrophenyl & 2-hydroxy-3,5-dichlorophenyl & $>6.25$ & 64 \\
$\mathbf{3 m}$ & 4-nitrophenyl & 3,4-dichlorophenyl & $>6.25$ & 59 \\
3n & 4-nitrophenyl & 3-phenoxyphenyl & $>6.25$ & 80 \\
\hline Rifampicin & - & - & 0.25 & 98 \\
\hline
\end{tabular}

Table 3. QikProp estimated drug-likeness parameters of compounds 3a-n.

\begin{tabular}{ccccccc}
\hline Molecule & SASA & QPlogBB & QPPMDCK & HOA\% & PSA & RO5 \\
\hline 3a & 646.79 & -0.47 & 4137.51 & 100 & 69.43 & 0 \\
3b & 682.19 & -0.50 & 4758.81 & 100 & 69.78 & 0 \\
3c & 767.89 & -0.44 & 6026.98 & 100 & 68.64 & 1 \\
3d & 737.19 & -0.01 & 10000 & 100 & 48.68 & 1 \\
3e & 750.03 & -0.35 & 10000 & 100 & 66.75 & 1 \\
3f & 735.48 & 0.10 & 10000 & 100 & 47.58 & 1 \\
3g & 739.18 & 0.13 & 10000 & 100 & 46.97 & 1 \\
3h & 773.05 & -0.47 & 5846.08 & 100 & 66.16 & 1 \\
3i & 852.83 & -0.46 & 2461.06 & 100 & 54.40 & 1 \\
3j & 614.58 & 0.01 & 1941.58 & 97.28 & 35.47 & 1 \\
3k & 778.90 & -0.53 & 5834.65 & 100 & 74.37 & 1 \\
31 & 780.69 & -1.69 & 564.20 & 82.61 & 111.84 & 1 \\
3m & 770.34 & -1.18 & 1273.97 & 93.04 & 91.97 & 1 \\
3n & 861.75 & -1.73 & 247.07 & 96.99 & 99.44 & 1 \\
\hline
\end{tabular}

\section{MATERIALS AND METHODS}

\subsection{Chemistry}

All chemicals were purchased locally from Aldrich, Fluka and Merck. Melting points were determined by SMP II melting point apparatus and uncorrected. FT-IR spectra were obtained on a Schimadzu FTIR-8400S spectrophotometer. ${ }^{1} \mathrm{H}-\mathrm{NMR}$ spectra were run on a Bruker Avance DPX-400 spectrometer in DMSO- $d_{6}$ with tetramethylsilane as the internal standard and all chemical shifts were reported as $\delta$ (ppm) values. Mass spectra were obtained with an Agilent 1100 MSD spectrometer. Elemental analyses were performed with Leco CHNS 932.

In light of our previous studies, ethyl 4-(benzoylamino)benzoate, 4-(benzoylamino)benzoyl hydrazide, 1-aroyl-4-alkyl/arylthiosemicarbazides and 2-alkyl/arylamino-5-(4-aminophenyl)-1,3,4-thiadiazoles were prepared $[18,29]$. 
4.1.2. General procedure for the preparation of 2-substitutedamino-5-[4-substitutedbenzylideneaminophenyl]-1,3,4thiadiazoles $(3 a-n)$

Compounds $2(0.015 \mathrm{~mol})$ were treated with equimolar amount of aromatic aldehydes in anhydrous methanol. The resulting mixture was heated under reflux for $45 \mathrm{~min}$. After cooling to room temperature, the resulting products were filtered and washed with warm ethanol [22].

2-Methylamino-5-[4-(2-hydroxy-3,5-dichlorobenzylideneamino)phenyl]-1,3,4-thiadiazole (3a). Yield: 37\%;

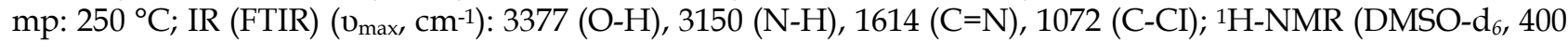
$\mathrm{MHz}) \delta(\mathrm{ppm}): 2.85$ (s, 3H, CH $), 7.39-7.88$ (m, 6H, Ar-H), 8.98 (s, 1H, -N=C-H), 14.11 (s, 1H, -OH); APCI Pos m/z $380\left(\mathrm{M}+\mathrm{H}^{+}, 8 \%\right), 79.1$ (100\%). Anal. Calcd for $\mathrm{C}_{16} \mathrm{H}_{12} \mathrm{Cl}_{2} \mathrm{FN}_{4} \mathrm{OS}$ (379.26): C, 50.67; H, 3.19; N, $14.77 ;$ S, 8.45. Found: C, 50.89; H, 3.70; N, 14.88; S, 8.52.

2-Ethylamino-5-[4-(2-hydroxy-3,5-dichlorobenzylideneamino)phenyl]-1,3,4-thiadiazole (3b). Yield: 79\%;

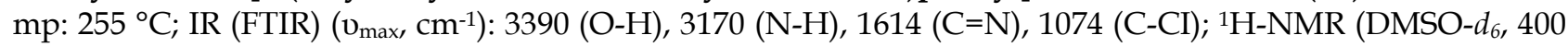
$\mathrm{MHz}) \delta(\mathrm{ppm}) 1.21\left(\mathrm{t}, 3 \mathrm{H}, \mathrm{CH}_{3}\right), 3.37\left(\mathrm{q}, 2 \mathrm{H}, \mathrm{CH}_{2}\right), 7.58-7.89(\mathrm{~m}, 6 \mathrm{H}, \mathrm{Ar}-\mathrm{H}), 8.01(\mathrm{t}, 1 \mathrm{H},-\mathrm{NH}$ of thiadiazole), 9.09 (s, 1H, -N=C-H), 14.24 (s, 1H, -OH); APCI Pos m/z $394\left(\mathrm{M}+\mathrm{H}^{+}, 4 \%\right), 221.0(100 \%)$. Anal. Calcd for $\mathrm{C}_{17} \mathrm{H}_{14} \mathrm{Cl}_{2} \mathrm{~N}_{4} \mathrm{OS}$ (393.29): C, 51.92; H, 3.59; N, 14.25; S, 8.15. Found: C, 52.51; H, 4.00; N, 14.53; S, 8.21.

2-Cyclohexylamino-5-[4-(2-hydroxy-3,5-dichlorobenzylideneamino)phenyl]-1,3,4-thiadiazole (3c). Yield: 72\%; mp: 241-243 ${ }^{\circ} \mathrm{C}$; IR (FTIR) $\left(\mathrm{v}_{\max }, \mathrm{cm}^{-1}\right): 3300(\mathrm{O}-\mathrm{H}), 3179$ (N-H), 1614 (C=N), 1082 (C-CI); ${ }^{1} \mathrm{H}-\mathrm{NMR}$ (DMSO$\left.d_{6}, 400 \mathrm{MHz}\right) \delta(\mathrm{ppm}): 1.11-2.09\left(\mathrm{~m}, 10 \mathrm{H}\right.$, cyclohexyl $\left.\mathrm{CH}_{2}\right), 3.57(\mathrm{~s}, 1 \mathrm{H}, \mathrm{CH}), 7.57-7.99(\mathrm{~m}, 7 \mathrm{H}, \mathrm{Ar}-\mathrm{H}$ and NH), 9.09 (s, 1H, -N=C-H), 14.40 (s, 1H, -OH); APCI Pos m/z $448\left(\mathrm{M}+\mathrm{H}^{+}, 29 \%\right), 275.1$ (100\%); Anal. Calcd for $\mathrm{C}_{21} \mathrm{H}_{20} \mathrm{Cl}_{2} \mathrm{~N}_{4} \mathrm{OS}$ (447.38): C, 56.38; H, 4.51; N, 12.52; S, 7.17. Found: C, 56.78; H, 4.62; N, 12.79; S, 7.15.

2-Benzylamino-5-[4-(2,4-dichlorobenzylideneamino)phenyl]-1,3,4-thiadiazole (3d). Yield: 64\%; mp: 260-263 ${ }^{\circ} \mathrm{C}$; IR (FTIR) ( $\left.\mathrm{v}_{\max }, \mathrm{cm}^{-1}\right): 3194(\mathrm{~N}-\mathrm{H}), 1614(\mathrm{C}=\mathrm{N}), 1099$ (C-CI); APCI Pos m/z $440\left(\mathrm{M}+\mathrm{H}^{+}, 6 \%\right), 79.1$ (100\%); Anal. Calcd for $\mathrm{C}_{22} \mathrm{H}_{16} \mathrm{CI}_{2} \mathrm{~N}_{4} \mathrm{~S}$ (439.36): C, 60.14; H, 3.67; N, 12.75; S, 7.30. Found: C, 60.00; H, 3.84; N, 12.74; S, 7.18 .

2-(4-Fluorophenyl)amino-5-[4-(2-hydroxy-3,5-dichlorobenzylideneamino)phenyl]-1,3,4-thiadiazole (3e).

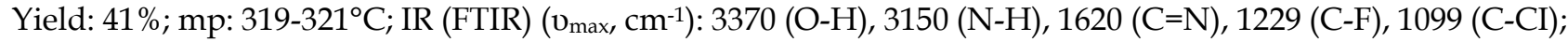
${ }^{1} \mathrm{H}-\mathrm{NMR}\left(\mathrm{DMSO}-d_{6}, 400 \mathrm{MHz}\right) \delta$ (ppm) 7.23 (t, 2H, ortho-protons to F), 7.41-8.09 (m, 8H, Ar-H), 9.11 (s, $1 \mathrm{H},-$

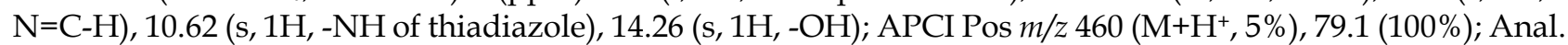
Calcd for $\mathrm{C}_{21} \mathrm{H}_{13} \mathrm{Cl}_{2} \mathrm{FN}_{4} \mathrm{OS}$ (459.32): C, 54.91; H, 2.85; N, 12.20; S, 6.98. Found: C, 54.92; H, 3.71; N, $12.43 ; \mathrm{S}, 7.09$.

2-(4-Fluorophenyl)amino-5-[4-(2,4-dichlorobenzylideneamino)phenyl]-1,3,4-thiadiazole (3f). Yield: 86\%;

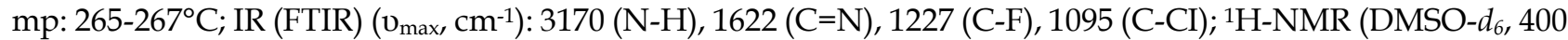
$\mathrm{MHz}) \delta(\mathrm{ppm}) 7.22(\mathrm{t}, 2 \mathrm{H}$, ortho-protons to $\mathrm{F}), 7.44(\mathrm{~d}, 2 \mathrm{H}$, ortho-protons to imine, $J=8.5 \mathrm{~Hz}), 7.58\left(\mathrm{dd}, 1 \mathrm{H}, \mathrm{C}_{6}\right.$ proton of 2,4-dichlorophenyl ring, $J=2.0 \mathrm{~Hz}, J=8.5 \mathrm{~Hz}$ ), 7.64-7.77 (dd, 2H, meta-protons to $\mathrm{F}, J=4.7 \mathrm{~Hz}, J=9.0$ $\mathrm{Hz}), 7.82\left(\mathrm{~d}, 1 \mathrm{H}, \mathrm{C}_{3}\right.$ proton of 2,4-dichlorophenyl ring, $\left.J=2.0 \mathrm{~Hz}\right), 7.95(\mathrm{~d}, 2 \mathrm{H}$, meta-protons to imine, $J=8.4 \mathrm{~Hz})$, $8.22\left(\mathrm{~d}, 1 \mathrm{H}, \mathrm{C}_{5}\right.$ proton of 2,4-dichlorophenyl ring, J= $\left.8.50 \mathrm{~Hz}\right), 8.87(\mathrm{~s}, 1 \mathrm{H},-\mathrm{N}=\mathrm{C}-\mathrm{H}), 10.59(\mathrm{~s}, 1 \mathrm{H},-\mathrm{NH}$ of thiadiazole); APCI Pos m/z $444\left(\mathrm{M}+\mathrm{H}^{+}, 13 \%\right)$, 474.9 (100\%); Anal. Calcd for $\mathrm{C}_{21} \mathrm{H}_{13} \mathrm{C}_{12} \mathrm{FN}_{4} \mathrm{~S}$ (443.32): C, 56.89; H, 2.96; N, 12.64; S, 7.23. Found: C, 57.33; H, 2.74; N, 12.97; S, 7.29.

2-(4-Fluorophenyl)amino-5-[4-(3,4-dichlorobenzylideneamino)phenyl]-1,3,4-thiadiazole (3g). Yield: 86\%; mp: $272-275{ }^{\circ} \mathrm{C}$; IR (FTIR) $\left(\mathrm{v}_{\max }, \mathrm{cm}^{-1}\right): 3150(\mathrm{~N}-\mathrm{H}), 1620(\mathrm{C}=\mathrm{N}), 1227$ (C-F), 1095 (C-CI); ${ }^{1} \mathrm{H}-\mathrm{NMR}$ (DMSO-d ${ }_{6}$ $400 \mathrm{MHz}) \delta(\mathrm{ppm}) 7.22(\mathrm{t}, 2 \mathrm{H}$, ortho-protons to $\mathrm{F}), 7.43(\mathrm{~d}, 2 \mathrm{H}$, ortho-protons to imine, $J=8.51 \mathrm{~Hz}), 7.58-7.77(\mathrm{~m}$, $2 \mathrm{H}$, meta-protons to $\mathrm{F}), 7.82\left(\mathrm{~d}, 1 \mathrm{H}, \mathrm{C}_{6}\right.$ proton of 3,4-dichlorophenyl ring), 7.86-8.04 (m, 3H, meta-protons to imine and $\mathrm{C}_{5}$ proton of 2,4-dichlorophenyl ring), $8.18\left(\mathrm{~s}, 1 \mathrm{H}, \mathrm{C}_{2}\right.$ proton of 3,4-dichlorophenyl ring), $8.72(\mathrm{~s}, 1 \mathrm{H}$, -N=C-H), 10.58 (s, 1H, -NH of thiadiazole); APCI Pos m/z $444\left(\mathrm{M}+\mathrm{H}^{+}, 7 \%\right), 287.0$ (100\%); Anal. Calcd for $\mathrm{C}_{21} \mathrm{H}_{13} \mathrm{C}_{12} \mathrm{FN}_{4} \mathrm{~S}$ (443.32): C, 56.89; H, 2.96; N, 12.64; S, 7.23. Found: C, 57.16; H, 3.24; N, 13.11; S, 7.25.

2-(4-Methylphenyl)amino-5-[4-(2-hydroxy-3,5-dichlorobenzylideneamino)phenyl]-1,3,4-thiadiazole (3h).

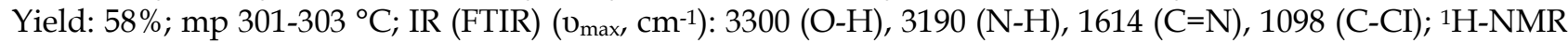
$\left(\mathrm{DMSO}_{6}, 400 \mathrm{MHz}\right) \delta(\mathrm{ppm}): 2.24\left(\mathrm{~s}, 3 \mathrm{H}, \mathrm{CH}_{3}\right), 6.63-7.99(\mathrm{~m}, 10 \mathrm{H}, \mathrm{Ar}-\mathrm{H}), 9.81(\mathrm{~s}, 1 \mathrm{H},-\mathrm{N}=\mathrm{C}-\mathrm{H}), 10.52$ (s, $1 \mathrm{H},-$ $\mathrm{NH}$ of thiadiazole), $14.22(\mathrm{~s}, 1 \mathrm{H},-\mathrm{OH}) ;$ APCI Pos $m / z 456\left(\mathrm{M}+\mathrm{H}^{+}, 2 \%\right), 283.0$ (100\%); Anal. Calcd for $\mathrm{C}_{22} \mathrm{H}_{16} \mathrm{Cl}_{2} \mathrm{~N}_{4} \mathrm{OS}$ (455.35): C, 58.16; H, 3.33; N, 12.33; S, 7.06. Found: C, 58.00; H, 3.23; N, 11.55; S, 7.05.

2-(4-Methylphenyl)amino-5-[4-(2,4-dichlorobenzylideneamino)phenyl]-1,3,4-thiadiazole (3i). Yield: 78\%;

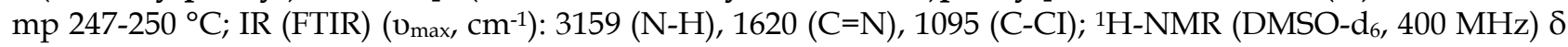


(ppm): 2.31 (s, 3H, $\left.\mathrm{CH}_{3}\right), 7.02-8.21$ (m, 11H, Ar-H), 8.87, 8.89 (2s, 1H, -N=C-H), 10.46 (s, 1H, -NH of thiadiazole); APCI Pos $m / z 440\left(\mathrm{M}+\mathrm{H}^{+}, 21 \%\right), 283.0(100 \%)$; Anal. Calcd for $\mathrm{C}_{22} \mathrm{H}_{16} \mathrm{C}_{12} \mathrm{~N}_{4} \mathrm{~S}$ (439.36): C, 60.14; $\mathrm{H}, 3.67 ; \mathrm{N}, 12.75$; S, 7.30. Found: C, 60.31; H, 3.47; N, 13.09; S, 7.39.

2-(4-Methylphenyl)amino-5-[4-(3-(4-phenoxy))benzylideneaminophenyl]-1,3,4-thiadiazole (3j). Yield: 47 \%; mp 218-220 ${ }^{\circ} \mathrm{C}$; IR (FTIR) $\left(\mathrm{v}_{\max }, \mathrm{cm}^{-1}\right): 3178(\mathrm{~N}-\mathrm{H}), 1617(\mathrm{C}=\mathrm{N}) ;{ }^{1} \mathrm{H}-\mathrm{NMR}$ (DMSO- $\left.d_{6}, 400 \mathrm{MHz}\right) \delta(\mathrm{ppm}) 2.29(\mathrm{~s}$, $\left.3 \mathrm{H},-\mathrm{CH}_{3}\right), 7.07-7.99(\mathrm{~m}, 17 \mathrm{H}, \mathrm{Ar}-\mathrm{H}), 8.69(\mathrm{~s}, 1 \mathrm{H},-\mathrm{N}=\mathrm{C}-\mathrm{H}), 10.44(\mathrm{~s}, 1 \mathrm{H},-\mathrm{NH}$ of thiadiazole); APCI Pos $\mathrm{m} / z 463$ $\left(\mathrm{M}+\mathrm{H}^{+}, 4 \%\right), 283.0(100 \%)$; Anal. Calcd for $\mathrm{C}_{28} \mathrm{H}_{22} \mathrm{~N}_{4} \mathrm{OS}$ (462.56): $\mathrm{C}, 72.70 ; \mathrm{H}, 4.79 ; \mathrm{N}, 12.11 ; \mathrm{S}, 6.93$. Found: $\mathrm{C}$, 72.46; H, 4.62; N, 12.51; S, 6.93.

2-(4-Methoxyphenyl)amino-5-[4-(2-hydroxy-3,5-dichlorobenzylideneamino)phenyl]-1,3,4-thiadiazole (3k). Yield 55\%; mp 277 ${ }^{\circ}$; IR (FTIR) ( $\left.\mathrm{v}_{\max }, \mathrm{cm}^{-1}\right)$ : $3370(\mathrm{O}-\mathrm{H}), 3150(\mathrm{~N}-\mathrm{H}), 1614$ (C=N), 1080 (C-CI); ${ }^{1} \mathrm{H}-\mathrm{NMR}$ (DMSO$\left.d_{6}, 400 \mathrm{MHz}\right) \delta(\mathrm{ppm}) 3.77\left(\mathrm{~s}, 3 \mathrm{H}, \mathrm{CH}_{3}\right), 6.63-8.08(\mathrm{~m}, 10 \mathrm{H}, \mathrm{Ar}-\mathrm{H}), 9.10(\mathrm{~s}, 1 \mathrm{H},-\mathrm{N}=\mathrm{C}-\mathrm{H}), 10.39(\mathrm{~s}, 1 \mathrm{H},-\mathrm{NH}$ of thiadiazole), $14.21(\mathrm{~s}, 1 \mathrm{H},-\mathrm{OH})$; APCI Pos $m / z 472\left(\mathrm{M}+\mathrm{H}^{+}, 1 \%\right), 299(100 \%)$; Anal. Calcd for $\mathrm{C}_{22} \mathrm{H}_{16} \mathrm{Cl}_{2} \mathrm{~N}_{4} \mathrm{O}_{2} \mathrm{~S}$ (471.36): C, 56.06; H, 3.42; N, 11.89; S, 6.80. Found: C, 55.73; H, 3.20; N, 11.90; S, 6.55.

2-(4-Nitrophenyl)amino-5-[4-(2-hydroxy-3,5-dichlorobenzylideneamino)phenyl]-1,3,4-thiadiazole (31). Yield 80\%; mp 343-345 ${ }^{\circ} \mathrm{C}$; IR (FTIR) ( $\left.\mathrm{v}_{\max }, \mathrm{cm}^{-1}\right): 3340(\mathrm{O}-\mathrm{H}), 3200(\mathrm{~N}-\mathrm{H}), 1643(\mathrm{C}=\mathrm{N}), 1077$ (C-CI); ${ }^{1} \mathrm{H}-\mathrm{NMR}$ $\left(\right.$ DMSO- $\left._{6}, 400 \mathrm{MHz}\right) \delta(\mathrm{ppm}):$ 7.23-8.44 (m, 10H, Ar-H), 10.53 (s, 1H, -N=C-H), 11.29 (br.s, 1H, -NH of thiadiazole); APCI Pos $m / z 487\left(\mathrm{M}+\mathrm{H}^{+}, 2 \%\right), 417.9(100 \%)$; Anal. Calcd for $\mathrm{C}_{21} \mathrm{H}_{13} \mathrm{Cl}_{2} \mathrm{~N}_{5} \mathrm{O}_{3} \mathrm{~S}$ (486.33): C, 51.86; H, 2.69; N, 14.40; S, 9.87. Found: C, 51.78; H, 2.70; N, 14.37; S, 9.87.

2-(4-Nitrophenyl)amino-5-[4-(3,4-dichlorobenzylideneamino)phenyl]-1,3,4-thiadiazole (3m). Yield 61\%; mp $345^{\circ} \mathrm{C}$; IR (FTIR) ( $\left.\mathrm{v}_{\max }, \mathrm{cm}^{-1}\right)$ : $3390(\mathrm{O}-\mathrm{H}), 3259(\mathrm{~N}-\mathrm{H}), 1643(\mathrm{C}=\mathrm{N}), 1074$ (C-CI); ${ }^{1} \mathrm{H}-\mathrm{NMR}$ (DMSO-d $\left.d_{6}, 400 \mathrm{MHz}\right)$ $\delta$ (ppm): 7.29-8.50 (m, 11H, Ar-H), $10.53(\mathrm{~s}, 1 \mathrm{H},-\mathrm{N}=\mathrm{C}-\mathrm{H}), 11.18$ (br.s, $1 \mathrm{H},-\mathrm{NH}$ of thiadiazole); APCI Pos m/z $471\left(\mathrm{M}+\mathrm{H}^{+}, 1 \%\right), 418.0(100 \%)$. Anal. Calcd for $\mathrm{C}_{21} \mathrm{H}_{13} \mathrm{C}_{12} \mathrm{~N}_{5} \mathrm{O}_{2} \mathrm{~S} .3 \mathrm{H}_{2} \mathrm{O}$ (524.37): C, 48.10; $\mathrm{H}, 3.65 ; \mathrm{N}, 13.36 ; \mathrm{S}, 6.11$. Found: C, 47.75; H, 3.93; N, 13.73; S, 6.75 .

2-(4-Nitrophenyl)amino-5-[4-(3-(4-phenoxy))benzylideneaminophenyl]-1,3,4-thiadiazole (3n). Yield: 60\%; mp 243-245 ${ }^{\circ} \mathrm{C}$. IR (FTIR) $\left(\mathrm{v}_{\max }, \mathrm{cm}^{-1}\right): 3350(\mathrm{O}-\mathrm{H}), 3150(\mathrm{~N}-\mathrm{H}), 1614(\mathrm{C}=\mathrm{N}) ;{ }^{1} \mathrm{H}-\mathrm{NMR}$ (DMSO- $\left.d_{6}, 400 \mathrm{MHz}\right) \delta$ (ppm): 6.98-8.38 (m, 17H, Ar-H), 8.69 (s, 1H, -N=C-H), 11.32 (br.s, $1 \mathrm{H},-\mathrm{NH}$ of thiadiazole); APCI Pos m/z 495 $\left(\mathrm{M}+\mathrm{H}^{+}, 36 \%\right)$, $314.0(100 \%)$; Anal. Calcd for $\mathrm{C}_{27} \mathrm{H}_{19} \mathrm{~N}_{5} \mathrm{O}_{3} \mathrm{~S} .4 \mathrm{H}_{2} \mathrm{O}$ (565.59): C, 57.34; H, 4.81; N, 12.38; S, 5.67. Found: C, 57.59; H, 4.15; N, 12.48; S, 5.93 .

\subsection{Antituberculosis activity}

All of the compounds were evaluated for their in vitro antituberculosis activity against Mycobacterium tuberculosis H37Rv by Tuberculosis Activity Antimicrobial Acquisition and Coordinating Facility (TAACF) of Southern Research. Primary screening was conducted at $6.25 \mu \mathrm{g} \mathrm{ml}^{-1}$ against Mycobacterium tuberculosis H37Rv in BACTEC 12B medium using broth microdilution assay [30].

\subsection{Computational analysis}

\subsubsection{QikProp analysis}

Three-dimensional (3D) structure of all aforementioned ligands were sketched in Chem Draw ultra 12.0.2.1076 and imported to LigPrep module of Schrödinger [31]. In LigPrep, ionization state of each compound was maintained at $\mathrm{pH} 7.0 \pm 2$ and for each ligand, tautomer's were also generated [32]. Computational quantitative assessment of drug likeliness for each compound was also performed which includes multiple parameters related to Lipinski's Rule of five (RO5) criteria, absorption, distribution, metabolism and excretion (ADME) were calculated using QikProp. Molecular descriptors calculated in the current study are SASA, QPPMDCK, HOA\%, QPlogBB and PSA to provide insight into hydrophobicity, lipophilicity and metabolic properties of the compounds under study [33, 34]. 
Acknowledgements: The authors are thankful to Dr Joseph A. Maddry from the Tuberculosis Antimicrobial Acquisition and Coordination Facility (TAACF) for the in vitro evaluation of antimycobacterial activity using Mycobacterium tuberculosis H37Rv. The authors would like to thank to Assoc. Prof. Dr. Abdulilah Ece from Biruni University for his generous help at the computational studies. This study was supported by Marmara University Scientific Research Project Commission, Project number: SAG-103/081004.

Author contributions: Concept - S.T., S.K., E.E.O.E.; Design - S.T., S.K., E.E.O.E.; Supervision - S.K.; Resources - S.T., S.K., A.M.; Materials - S.T., S.K., A.M.; Data Collection and/or Processing - S.T., S.K., A.M.; Analysis and/or Interpretation - S.T., S.K., A.M.; Literature Search - S.T., S.K., A.M.; Writing - S.T., S.K., A.M.; Critical Reviews - S.T., S.K., A.M., E.E.O.E.

Conflict of interest statement: The authors declared no conflict of interest.

\section{REFERENCES}

[1] Rawat P, Singh RN, Niranjan P, Ranjan A, Holguin NRF. Evaluation of antituberculosis activity and DFT study ondipyrromethane-derived hydrazone derivatives. J Mol Struct. 2017; 1149: 539-548. [CrossRef]

[2] Clark MP, Wang T, Perola E, Deininger DD, Zuccola HJ, Jones SM, Gao H, VanderVen BC, Russel DG, Shoen CM, Cynamon MH, Thompson JA, Locher CP. 2-N-Arylthiazole inhibitors of Mycobacterium tuberculosis. Bioorg Med Chem Lett. 2017; 27: 3987-3991. [CrossRef]

[3] WHO Global tuberculosis report 2019. http://www.who.int/tb/publications/global_report/en/ (accessed on 14 July 2020).

[4] Mandewale MC, Patil UC, Shedge SV, Dappadwad UR, Yamgar RS. A review on quinoline hydrazone derivatives as a new class of potent antitubercular and anticancer agents. Beni-Suef Univ J Basic Appl Sci. 2017; 6: 354-361. [CrossRef]

[5] Velezheva V, Brennan P, Ivanov P, Kornienko A, Lyubimov S, Kazarian K, Nikonenko B, Majorov K, Apt A. Synthesis and antituberculosis activity of indole-pyridine derived hydrazides, hydrazide-hydrazones, and thiosemicarbazones. Bioorg Med Chem Lett. 2016; 26: 978-985. [CrossRef]

[6] Pawaiya A, Pawaiya P, Rajput S, Agrawal MC, Bhatnagar RK. Synthesis, characterization and antifungal activity of hydrazone Schiff base. Int J Curr Res. 2014; 6(9): 8423-8426.

[7] Al-Masoudi WA, Al-Tememy TM, Al-Assadi RH. Computational study and antimicrobial activity of few Dapsone Schiff base derivatives. Eur J Chem. 2014; 5(2): 351-355. [CrossRef]

[8] Batra N, Batra S, Aggarwal N, Nagori BP. Schiff bases as CNS active agents: a review. Am J PharmTech Res. 2013; 3(4): 1-19.

[9] Ejidike IP, Ajibade PA. Transition metal complexes of symmetrical and asymmetrical Schiff bases as antibacterial, antifungal, antioxidant, and anticancer agents: progress and prospects. Rev Inorg Chem 2015; 35(4): 191-224. [CrossRef]

[10] Nath M, Saini PK, Kumar A. New di- and triorganotin (IV) complexes of tripodal Schiff base ligand containing three imidazole arms: Synthesis, structural characterization, anti-inflammatory activity and thermal studies. J Organomet Chem. 2010; 695: 1353-1362. [CrossRef]

[11] Zoubi WA, Al-Hamdani AAS, Kaseem M. Synthesis and antioxidant activities of Schiff bases and their complexes: a review. Appl Organomet Chem. 2016; 30: 810-817. [CrossRef]

[12] Baran NY, Karakışla M, Demir HÖ, Saçak M. Synthesis, characterization, conductivity and antimicrobial study of a novel thermally stable polyphenol containing azomethine group. J Mol Struct. 2016; 1123: 153-161. [CrossRef]

[13] Matysiak J. Biological and pharmacological activities of 1,3,4-thiadiazole based compounds. Mini Rev Med Chem. 2015; 15(9): 762-775. [CrossRef]

[14] Pandey A, Dewangan D, Verma S, Mishra A, Dubey RD. Synthesis of Schiff bases of 2-amino-5-aryl-1,3,4-thiadiazole and its analgesic, antiinflammatory, anti-bacterial and antitubercular activity. Int J ChemTech Res. 2011; 3(1): 178184 .

[15] Hwang TJ, Wares DF, Jafarov A, Jakubowiak W, Nunn P, Keshavjee S. Safety of cycloserine and terizidone for the treatment of drug-resistant tuberculosis: a meta-analysis. Int J Tuberc Lung Dis. 2013; 17(10): 1257-1266. [CrossRef]

[16] Foroumadi A, Kiani Z, Soltani F. Antituberculosis agents VIII. Synthesis and in vitro antimycobacterial activity of alkyl alpha-[5-(5-nitro-2-thienyl)-1,3,4-thiadiazole-2-ylthio]acetates. Farmaco. 2003; 58: 1073-1076. [CrossRef] 
[17] Kolavi G, Hegde V, Khazi IA, Gadad P. Synthesis and evaluation of antitubercular activity of imidazo[2,1b] $[1,3,4]$ thiadiazole derivatives. Bioorg Med Chem 2006; 14: 3069-3080. [CrossRef]

[18] Karakuş S, Rollas S. Synthesis and antimycobacterial activity of some 2-(4-aminophenyl)- 5-substituted amino-1,3,4thiadiazole derivatives and their coupling products. Marmara Pharm J. 2016; 20: 199-206. [CrossRef]

[19] Chitra S, Paul N, Muthusubramanian S, Manisankar P, Yogeeswari P, Sriram D. Synthesis of 3heteroarylthioquinoline derivatives and their in vitro antituberculosis and cytotoxicity studies. Eur J Med Chem. 2011; 46: 4897-4903. [CrossRef]

[20] Oruc EE, Rollas S, Kandemirli F, Shvets N, Dimoglo AS. 1,3,4-Thiadiazole derivatives. Synthesis, structure elucidation, and structure-antituberculosis activity relationship investigation. J Med Chem. 2004; 47: 6760-6767. [CrossRef]

[21] Tatar E, Küçükgüzel ŞG, Karakuş S, De Clercq E, Andrei G, Snoeck R, Pannecouque C, Öktem Okullu S, Ünübol N, Kocagöz T, Kalaycı S, Şahin F, Küçükgüzel İ. Synthesis and biological evaluation of some new 1,3,4-thiadiazole and 1,2,4-triazole derivatives from $L$-methionine as antituberculosis and antiviral agents. Marmara Pharm J. 2015; 19: 88102. [CrossRef]

[22] Küçükgüzel ŞG, Küçükgüzel İ, Tatar E, Rollas S, Şahin F, Güllüce M, Clercq ED, Kabasakal L. Synthesis of some novel heterocyclic compounds derived from diflunisal hydrazide as potential anti-infective and anti-inflammatory agents. Eur J Med Chem. 2007; 42: 893-901. [CrossRef]

[23] Solak N, Rollas S. Synthesis and antituberculosis activity of 2-(aryl/alkylamino)-5-(4-aminophenyl)-1,3,4thiadiazoles and their Schiff bases. ARKIVOC. 2006; 12: 173-181. [CrossRef]

[24] Suleymanoglu N, Ustabas R, Alpaslan YB, Coruh U, Karakus S, Rollas S. 2-Propylamino-5-[4-(2-hydroxy-3,5dichlorobenzylideneamino) phenyl]-1,3,4-thiadiazole: X-ray and DFT-calculated structures. Struct Chem. 2010; 21: 59-65. [CrossRef]

[25] Güngör Ö, Gürkan P. Synthesis and spectroscopic properties of novel asymmetric Schiff bases. Spectrochim Acta Mol Biomol Spectros. 2010; 77: 304-311. [CrossRef]

[26] Tahtaci H, Karacı H, Ece A, Er M, Şeker MG. Design, synthesis, SAR and molecular modeling studies of novel imidazo [2,1-b][1,3,4]thiadiazole derivatives as highly potent antimicrobial agents. Mol Inform. 2018; 37(3): 1700083. [CrossRef]

[27] Ece A. Towards more effective acetylcholinesterase inhibitors: a comprehensive modelling study based on human acetylcholinesterase protein-drug complex. J Biomol Struct Dyn. 2020; 38(2): 565-572. [CrossRef]

[28] Vistoli G, Pedretti A, Testa B. Assessing drug-likeness - what are we missing? Drug Discov Today. 2008; 13(7): 285294. [CrossRef]

[29] Karakus S, Rollas S. Synthesis and antituberculosis activity of new $N$-phenyl-N'-[4-(5-alkyl/arylamino-1,3,4thiadiazole-2-yl)phenyl]thioureas. Farmaco 2002; 57: 577-581. [CrossRef]

[30] Collins LA, Franzblau SG. Microplate alamar blue assay versus BACTEC 460 system for high-throughput screening of compounds against Mycobacterium tuberculosis and Mycobacterium avium. Antimicrob Agents Chemother. 1997; 41(5): 1004-1009.

[31] Schrödinger Release 2019-3: LigPrep, Schrödinger, LLC, New York, NY, 2019.

[32] Shelley JC, Cholleti A, Frye LL, Greenwood JR, Timlin MR, Uchimaya M. Epik: a software program for pKa prediction and protonation state generation for drug-like molecules. J Comput Aided Mol Des. 2007; 21 (12): 681-691. [CrossRef]

[33] Er M, Abounakhla AM, Tahtaci H, Bawah AH, Çınaroğlu SS, Onaran A, Ece A. An integrated approach towards the development of novel antifungal agents containing thiadiazole: synthesis and a combined similarity search, homology modelling, molecular dynamics and molecular docking study. Chem Cent J. 2018; 12 (1): 121. [CrossRef]

[34] Maryam A, Khalid RR, Siddiqi AR, Ece A. E-pharmacophore based virtual screening for identification of dual specific PDE5A and PDE3A inhibitors as potential leads against cardiovascular diseases. J Biomol Struct Dyn. 2020; 1-16. [CrossRef] 